\title{
DEEPEN THE GIS SPATIAL ANALYSIS THEORY STUDYING THROUGH THE GRADUAL PROCESS OF PRACTICE
}

\author{
Y.G. Yi ${ }^{\text {a b }}$, H.P. Liuc, *, X.P. Liu ${ }^{\mathrm{d}}$ \\ a School of Geography, Beijing Normal University,100875,Beijing,China-996521051@qq.com \\ b School of Geology and Geomatics, Tianjin Chengjian University,300384, Tianjin,China-yiyaoguo@sohu.com \\ c School of Geography, Beijing Normal University,100875,Beijing,China -hpliu@bnu.edu.cn \\ d School of Geography, Beijing Normal University,100875,Beijing,China - xpliu-l@qq.com
}

\section{Commission}

KEY WORDS: Procedural teaching method, Practice teaching, Spatial analysis, Innovation cultivation, Experiment operation modules, Theoretical knowledge.

\begin{abstract}
:
Spatial analysis is the key content of GIS basic theory course. In this paper, the importance of practice teaching for GIS spatial analysis theory studying and its implementation method are discussed combined with practice teaching arrangement of spatial analysis in the course "GIS theory and practice" based on the basic principle of procedural teaching theory and its teaching model. In addition, the concrete gradual practice process is mentioned in four aspects. By this way, the GIS spatial analysis theory studying can be deepened and the cultivation of students' comprehensive ability of Geography Science can be strengthened.
\end{abstract}

\section{INTRODUCTION}

Each subject has its regularity and characteristics as well as the rigorous logic system. If we want to systematically master, its study process is nothing that from shallow to deep, from simple to complex, from easy to difficult. Famous song dynasty theorist X.zhu said: "The law of reading is in the sequential and progressive", "not line on front, not step to behind, not understand this, not want that, so how, through gradual progress, the meaning determinate and the principle clear." It can clearly be seen ancients had concluded and handled that study law.

Procedural teaching method put forward by the famous American psychologist B.F. Skinner, refers to the knowledge of different subjects according to the intrinsic logic relation of the project is decomposed into a series of knowledge, which is continuity between projects and gradually deepened, and then let the students study each knowledge one by one in accordance with the order of the knowledge of project. With learning each project, students give feedback and reinforcement in a timely manner. Eventually they are able to grasp what they have learned and reach to the expected teaching objectives.

Spatial Analysis (SA) is the theory and method of spatial data analysis and model based on geographical objects' positions and morphological characteristics in the Geographic Information System (GIS) theoretical knowledge learning. It use various spatial analysis model and space operation to deep processing of spatial data in the GIS database, extract and find spatial information and discipline implied in the data. Learning "Spatial Analysis" course, students can master spatial correlation, inherent law and analysis algorithm widespread in spatial data and form the space thinking and analytical skills. Therefore, the spatial analysis is the core of the geographic information system and its importance is self-evident.

In this paper, how to exercise and improve the students' practical ability through definite practice teaching arrangement set up and implement is discussed based on the basic principle of procedural teaching theory and its teaching model, combined with spatial analysis theory teaching in the Geographic Information Systems Master course "GIS theory and practice". So, the students' comprehensive abilities could be strengthened and their professional qualities could be cultivated in order to undertake relevant project and the scientific research in the future.

\section{SETTING THE EXPERIMENTAL OPERATION MODULES OF DIFFERENT LEVELS AND ESTABLISHING THE SYSTEM OF PRACTICE TEACHING STEP BY STEP}

The key of procedural knowledge teaching is carefully setting up knowledge project sequences and strengthening practice teaching program procedures. Requires knowledge points will be design into a series of small problems with the organic links according to the procedures, and the degree of difficulty between various small problems gradually increases, but the difficulty increases slowly. By this way, the difficulty can be dispersed and each knowledge points can be handled while solving every small problem. Meanwhile, the procedures are in line with the principle of gradual and progressive approach, from easy to difficult, and enable students to understand knowledge connotations through practice teaching.

In the spatial analysis theory teaching of Master course "GIS theory and practice", we design and arrange eight practical programs according to different curriculum content and degree of difficulty. Eight GIS software operations by which practice teaching system of constitutes progressive approach can be constituted. We use two current mainstream GIS professional software in the experiments: "ArcGIS"@ ESRI, Inc. Copyright and "SuperMap"C SUPERMAP, Ctd. Copyright.

In the former six experiments, basic operations and spatial analysis methods of the most widely used international software

\footnotetext{
* Corresponding author. This is useful to know for communication with the appropriate person in cases with more than one author.
} 
"ArcGIS" are introduced, including the basic framework of software, spatial data collection and compiling, the establishment of spatial topological relations, vector and raster data spatial analysis methods and how to use "Model Builder" modules. In the latter two experiments, the data organization format, spatial visualization, image registration and basic spatial analysis procedures of the largest China software "SuperMap" are described. These eight practices are enable students to deepen the understanding of GIS spatial analysis connotations at each step of theoretical knowledge learning in the classroom.

\subsection{Basic Function Practices}

In order to give an overall concept of "ArcGIS" to students, the introduction of ESRI, Inc. and "ArcGIS" software components are presented and the function of "ArcMap", "ArcCatlog" and "Geoprocessing" modules in "ArcGIS Desktop" are highlighted in the experiment I. After the students have a general understanding of "ArcGIS", we specifically teaches digital operation experiments from which students can learn how to convert map elements into points, lines, polygons and how to accomplish simple data collection and property editing.

There are two main purpose of Geographic Information System: visualization and spatial analysis. Therefore, how to make map element layers into visualization and into thematic maps in "ArcGIS" is practiced in experiment II and experiment III. In experiment IV and experiment $\mathrm{V}$, the spatial analysis implementation process is detailed introduced in connection with the characteristics of vector and raster data.

Before making geographical phenomena into thematic maps, we set up the contents of GIS data operations in experiment II, including the geometry modification and attribute table operations of vector data, coordinate system determination and topological relations establishment. After the data is read into "ArcGIS", the work is to be carried out first to set the correct coordinate system and projection. Then, we require that students should master processing data and modifying data capabilities, such as road elements updating, copying and associating with the attribute table. The reason why we add content to familiar with the operation of GIS data is training students to accurately analyze the data sources coordinate location is correct or not and efficiently update data. So they will detours when using GIS data for spatial analysis in the future.

After completing experiment II, the GIS thematic map production practice of experiments III is following. We demand students marked their interested geographic features with legend symbols for the map can express a specific theme. Thematic map production is divided into two parts, one is symbolized and labeled with the data layers, and the other part is to add elements to thematic maps. Data layers can be separately displayed by these symbolized and labeled methods according to their property values. Adding elements refers to add auxiliary information such as compass, scale and legends surround the thematic map. After these two parts have been done, the above content is exported to a thematic map and this experiment is completed.

\subsection{Spatial Analysis Practices}

We set up two experiment procedures respectively according to different characteristics of vector and raster data and focus on vector data space analysis, road network analysis and Contour switch to TIN format methods in experiment IV based on its object-oriented and good topological features. For example, we establish a certain point and line buffers and obtain each vector element influence sphere in the analysis of urban housing selection experiment. Then, we integrated with all factors through overlay each layers. Finally, the optimal site of the regional house could be selected.

In the experiment $\mathrm{V}$, we focus on several raster data spatial analysis functions, such as distance cartographic, the cost and distance-weighted, data re-classification and multi-level merging based on its simply data structure and easily to simulate surface characteristics. For example, we transform all data of terrain, land use, influence degree of entertainment and existing schools distribution into raster data firstly when we analyze the optimal site of the regional school. Then we convert DEM elevation values into the slope data by slope generate tools in spatial analysis modules.

Then, we divide slope value into different levels using reclassification tools in the spatial analysis module according to relevant experience. The topography of adaptive hierarchical classification for school site can be determined.

Finally, the various factors are given different weights after a theoretical analysis, and then the most appropriate school address can be get by a grid weighted overlay analysis.

\subsection{Other Practices}

In the actual GIS project research and implementation process, the whole task is completed not just by a few simple GIS overlay operations function modules, usually through a multistep and multi-task to achieve. Meanwhile, many sub-tasks module would be called repeatedly. So, it is to say that the GIS projects are realized with the workflow feature which brings a certain degree of difficulty to build and optimize GIS system. In experiments VI, we practice how GIS workflow is achieved by "Model Builder" module in "ArcGIS". So the students could deeply understand the connotation of visual editing language and the character of GIS workflows by creating, editing and managing GIS model.

Experiment VII and VIII mainly describe the operation methods of "SuperMap" software to allow students have a certain understanding of the largest China GIS software and exercise their practical ability through specific examples. As "ArcGIS" and "SuperMap" have many similarities on the composition and function, we will follow the "ArcGIS" software learning methods and applied to the "SuperMap" studying in order to improve students' ability to comprehend by analogy. The main content of the experimental include the data organizational format of "SuperMap", spatial visualization, image registration and basic spatial analysis. Spatial analysis methods mainly comprise buffer analysis, overlay analysis, network analysis, and raster analysis. Through comparative analysis of the similarities and differences between "ArcGIS" and "SuperMap", the students' ability to learn the software could be deepened.

Through the above processes, we divide the entire practice teaching contents into eight closely related stages according logic system of the GIS discipline knowledge. The previous stage is the basis and bedding of the rear stage; the rear stage is the continuation and deepening of the previous stage. By this way, the students can take progress from unknown to known. With the student's knowledge level and cognitive level are promoting in turn, the degree of difficulty among various stages are gradually increasing. Thus, the students would subtly deepen the understanding of GIS spatial analysis theoretical knowledge in the practice operational processes due to the difference of the difficulty between the two phases is small. 


\section{HOW TO OPTIMIZE THE CONTENT OF} PRACTICE BY INTRODUCING THE RESEARCH CASE

\subsection{Theory Basis}

B.F.Skinner, the author of procedural teaching method, indicate that one should use continuous approach method and strengthen by the comprehensive ways finally if he want to achieve a very difficulty goal. Thus, he can achieve mastery from easy to difficult.

According to this theory, we abandon written or paper examination form in the past and carefully designed several natural phenomena distribution law research case in the final of "GIS theory and practice" curriculum. The students could choose one case to declare laws and analysis causes by GIS space analysis method and then present in the form of paper and PPT.

The case topics cover geography, hydrology, ecology and other aspects of the global environment according to the specialty of the students. The topic assign abide the principle combine random distribution and specialty preferred, which not only embodies the fairness in the problem distribution but also allow students to give full play in their professional knowledge. The data source of each case includes 2-4 thematic maps which related to the theme and a rule instruction to explain how to realize the theme. In order to prevent plagiarism, each allocated case subjects is not same.

We ask the students to separate into several groups to complete the assigned research case. Every member of the group has his own task and cooperates with other member in the solution process. Firstly, the group extract map elements would be used and hold the data source layer from the thematic maps. The steps include image registration, geographic coordinates and projection setting and topology error checking. Then, the group adds one to two types of data as the auxiliary data for a case study according to the designed ideas. After the data has been handled, the students need to find some references related with their cases and analysis the spatial distribution law and the formation cause of geographic phenomenon in theory. This procedure would cultivate students' ability of reading literature and analysis problems. After establish the flow chart, the students would present the theoretical law by "ArcGIS" spatial analysis method according to the accumulation of knowledge in the past and the exploration of new methods today.

\subsection{Case Analysis}

For example, there is a case about landslide and mud-rock flow disasters in China. The data sources which students achieved include 3 thematic maps about Chinese landslide and mud-rock flow geological disasters, which cover the disasters distribution around the whole country.

In order to find way to begin the research, group members need to find the relevant documents and understand the distribution of national disasters, the degree of risk, the contract of different region as well as specific reasons combined with thematic maps. After making a good approximate experimental program, they can conversion the geographic features into vector which are related to the case in the resource data firstly. Then, they search the relevant date on the Internet to offer an additional data source and constitute the raw experimental analysis data. In dealing with the raw data, they must keep the steps of setting geographical coordinates, inspecting topological relationship and entering attribute data to ensure data accuracy and lay the foundation for experimental analysis behind.
The group should use geological disasters data layer to make thematic map and "plug-speak" after handling raw data. As well as analysis of the disasters distribution and specific differences in the country, they could predict which factors trigger this geographic phenomenon based on theoretical knowledge.

After having data source and theoretical basis, group members find several factors led to the formation of geological disasters and the relationships between them through "ArcGIS" modeling function and establish theoretical model. The methods can be used include logistic regression, multiple linear regression and correlation matrix method. They ultimately get the relationship equation between hazards factors and geological disasters through a comprehensive analysis of the data characteristics and prove the guess according to the formula.

So far, Chinese distribution and intensity of landslide and mudrock flow geological disasters and their causes have been clearly illustrated by the experimental data through the data collation, scheme designing, data preparing, experimental analysis, and the final demonstration. As shown in Figure 1:

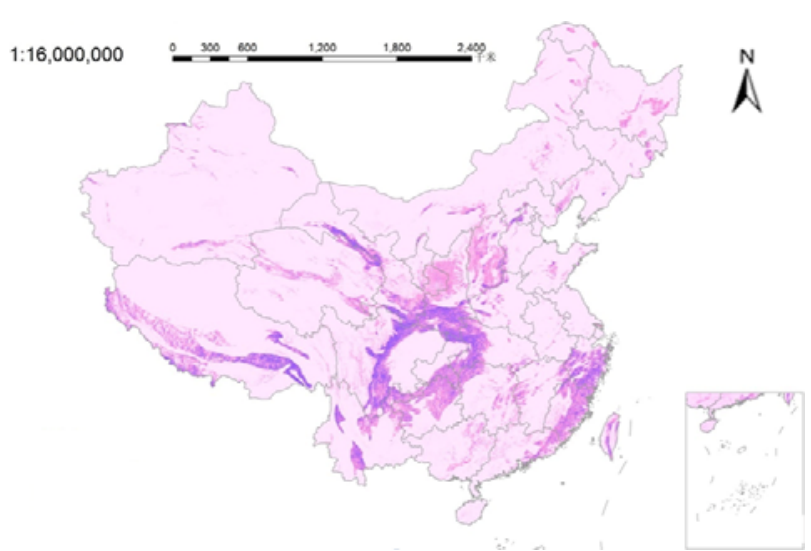

Figure 1 . The distribution and intensity of landslide and mudrock flow in China

We can see the final research case is summary and strengthened of the eight practices. From geographic features digitization to geography coordinates setting and from the spatial differentiation of inquiring to the specific cause analysis, every procedure requires students lay a solid foundation in the usual practices and have the ability to self-learning. Compared to written examination, this form could better test the students' understanding and self-innovation capability. In addition, the potential of students could be mined in maximize. From downloading thematic maps in internet to reporting similar to scientific project conclusion, this research case let students to learn how to use "ArcGIS" spatial analysis tools to find potential law from existing nature phenomena and realized a qualitative leap from deepen practical operation to truly understand the meaning of GIS spatial analysis theory.

\section{RICH IN THE FORM OF PRACTICE TEACHING TO FOSTER STUDENT' INNOVATION ABILITY}

A procedural teaching process must let students in an active learning state always. In other words, we should set up a variety of practical teaching methods for students to actively participate in the entire teaching process. Students are no longer listening and remembering passively, but thinking and solving problems initiatively. In this learning process, students become active participants instead of passive recipients. With the brains and 
hands be trained, the students' self-learning, independent study, and innovation abilities could be cultivated.

In specific aspects of the theoretical teaching and practical operation, the method of combination teacher-led teaching with students main body studying is used. The students operate GIS software led by the teacher in the classroom in order to eliminate the strangeness and overcome fear mentality. Then, the teacher arranges a little difficult homework to make students repeated the basic operation of proficiency in the classroom to enhance familiarity with the software. In the beginning of the next class, the teacher sets aside a few minutes on the more general question of the homework and gives a meticulous de compose and summary. So the students could gain new insights through reviewing old material. Such three practices method that learning in the classroom, practicing again at home, reviewing it again before next class, could emphasize the important and difficult points in studying repeatedly. Not only the students' memory is deepened, but also their active thinking is promoted and thus more knowledge points could be firmly grasped.

In order to increase the interaction opportunity between teacher and students and cultivate students' ability to think positively, we also open a special network FTP of the course and set a public WORD document "GIS Practice Course Highlights Issue" in it. All students can edit in this document. For example, put forward their own questions, answer other people's questions, even question and answer items themselves and record their experiences for all to share.

The teacher would particular answer the document questions regularly. This way is real-time and efficient for avoid taking class time. The form reflects the students as main body. Students become documents "writer" and documents become students' academic "microphone". A harmonious platform of equal to speak and a competitive environment of you struggle to catch my learning can be formed. Not only teacher, but also students could reap the study effect that can not get in the classroom.

The final research case is in the form of an open proposition. Each team can do the project from different views. This solving process requires analysis ideas are reasonable and final results are in line with the natural phenomena and laws. Each team needs to accumulate certain literatures reading about related case and think logically. It is a sublimation process from quantity to quality for students. Form of case can give students ample time to seek answers, stimulate their interests, challenge their abilities, and maximum excavate their potentials. Such cases have no standard answers, so that students can do them freely from their own views. The student's personality is full respected and their knowledge is sufficiently applied.

The above eight experiments is a fundamental part of the practice teaching. It aims to develop students' cognitive abilities of the GIS software. Students can study the basics knowledge and the basic operation of the GIS software. The final research cases give full play to students' ability to analyze and solve problems based on their theoretical knowledge and understanding of different topics. Their characteristics and creativity could be reflected in the cases. It is intended to exercise students' innovative thinking and academic ability. The repeated practices strengthen the students' practical operation capability; the open scientific cases cultivate students' creative thinking. The combination of these two methods can form a variety of teaching methods which are not tedious and can be achieved step by step. The object of "theory with reality and grow in practice" could be reached eventually.

\section{PERFECTING CURRICULUM EXAMINATION MODE AND EVALUATING OF TEACHING EFFECT SCIENTIFIC}

The final score of "GIS theory and practice" course is assessed comprehensively according to the results of the eight experiments and reports of the final research cases. The proportion of two parts accounted for $30 \%$ and $70 \%$. This scoring system will enable students to completely melt theoretical knowledge into practice operation. It is not only reflects the students' mastery of the knowledge, but also respect for individual differences of students. So the just and scientific of the score can be ensured.

In addition, we require students to submit their homework in uniform prescriptive time to ensure the principle fair and reasonable. If someone exceed this time, he need to submit it to FTP "Expired Job" folder in Internet. People who submit on schedule would be keep the score by full mark system, while people who submit overdue within a week would be scored by $50 \%$ mark system. If more delay or not to submit will not be scored. This way not only encourages students to complete assignments on time, and increases the rationality of score. Simultaneously, the students who cannot complete the job on schedule are given the opportunity to mend.

The assessment of the final research cases is in the form of students' public reply approach. The reply committee is composed of teachers and teaching assistants. The final score is comprehensive assessed by every number of committee. Each group is required to send a delegate to present their achievements in the form of PPT and the other groups are in the audience as observers. Each group finished, the respondent has time to answer public question. Both judges and attend groups can comment and ask questions to the respondent group. So that a geographic information science brainstorming can be formed between teachers and students in the process of questioning and answering, communicating with each other, questioning each other, exploring together, etc. The assessment mechanism put each group's outcome in public principle. It is open, transparent and fair.

After attending this form of reply, each group not only demonstrate their own achievements, but also learn thinking methods and the ways to solve problem from other groups. By this way, the students could deepen the understanding of theoretical knowledge through answering questions. Meanwhile, they could continue to strengthen and enhance their ability of logical thinking from others groups.

\section{CONCLUSIONS}

The procedural teaching method has unique charm in the actual teaching activities and profoundly effected on school education. It is emphasizes the learning process which is from easy to difficult and from simple to complex. It is consistent with the general rules and requirements for students learning. The experiences concluded in "GIS theory and practice" course demonstrate that this method not only improve teachers' efficiency to control the behavior of students, but also strengthen the students' ability to understand what they have learn through the gradual process of practice to deepen the GIS spatial analysis theory studying. It is indeed feasible and worth widely implementing in the teaching of colleges and universities, especially in geography subject. 


\section{REFERENCES}

A.R.Dang, Z. Liu, H.F. Jia., 2007.Exploration and practice on GIS teaching to application in universities. Geomatics World,2,pp.9-14.

B.P.Liang, Y.Li, H.Li.,2011. Investigate the remote sensing course teaching reform and practice of geography sciences in universities. Chinese Modern Educational Equipment,11, pp.112-114.

F.L.Bian., 1996. GIS principles and methods. Surveying and Mapping Press, Beijing ,pp. 111-121.

G.Q.Li, Q.Y.Liu, M.Deng,etc.,2009. On "Spatial Analysis" course teaching reform of surveying engineering specialty. Mine Surveying, 6,pp.87-89.

H.P.Liu, M.Bai, X.D.Mu., 2010.Practice student-centered teaching philosophy of earth science nternship.China University Teaching, 1, pp. 70-71.

J. F. Wang.,2006. Spatial Analysis. Science Press,Beijing, pp. 21-42.

L.S. Pi., 2004. Educational Psychology (third edition). Shanghai Education Press, Shanghai, pp. 321-332.

L.Wu, Y.Liu, J.Zhang,ect., 2001. Geographic information system principles, methods and applications, Science Press,Beijing, pp. 67-78.

Q.Xue.,2006.Reflections on geography practice teaching reform and innovation in higher normal school. Geography Education. 3, pp.75-76.

X.N. Huo.,2011. Procedural approach in teaching accounting .Economist, 2,pp.135-136.

X.Q.Yu, T.Zhang, Ji.S.Li.,2011. Research on "Geographic information system" course teaching methods of surveying engineering specialty. Geospatial information.2,pp.135-136.

\section{ACKNOWLEDGEMENT}

This study was supported by "Open Research Fund of State Key Laboratory of Information Engineering in Surveying, Mapping and Remote Sensing" (12 Important (02) ) - "Calculation and analysis of geographic based on spatial information services network". 